\title{
Frequency of actionable alterations in epidermal growth factor receptor (EGFR) wild type non-small cell lung cancer: experience of the Wide Catchment Area of Romagna (AVR)
}

\author{
Elisa Chiadini ${ }^{1}$, Matteo Canale ${ }^{1}$, Angelo Delmonte ${ }^{2}$, Claudio Dazzi ${ }^{3}$, Claudia Casanova ${ }^{3}$, Laura Capelli ${ }^{1}$, \\ Marita Mariotti ${ }^{2}$, Maximilian Papi ${ }^{4}$, Alessandro Gamboni ${ }^{5}$, Maurizio Puccetti ${ }^{6}$, Sara Bravaccini ${ }^{1}$, \\ Alessandra Dubini ${ }^{7}$, Daniele Calistri ${ }^{1}$, Lucio Crinò ${ }^{2}$, Paola Ulivi ${ }^{1}$ \\ ${ }^{1}$ Biosciences Laboratory, ${ }^{2}$ Department of Medical Oncology, Istituto Scientifico Romagnolo per lo Studio e la Cura dei Tumori (IRST) IRCCS, \\ Meldola, Italy; ${ }^{3}$ Medical Oncology Unit, S. Maria delle Croci Hospital, Ravenna, Italy; ${ }^{4}$ Oncology and Oncohematology Unit, Infermi Hospital, \\ Rimini, Italy; ${ }^{5}$ Medical Oncology Unit, Infermi Hospital, Faenza, Italy; ${ }^{6}$ Pathology Unit, S. Maria delle Croci Hospital, Ravenna, Italy; ${ }^{7}$ Pathology \\ Unit, Morgagni-Pierantoni Hospital, Forlì, Italy \\ Contributions: (I) Conception and design: P Ulivi; (II) Administrative support: None; (III) Provision of study materials or patients: M Puccetti, \\ A Dubini; (IV) Collection and assembly of data: A Delmonte, C Dazzi, C Casanova, M Mariotti, M Papi, A Gamboni; (V) Data analysis and \\ interpretation: P Ulivi; (VI) Manuscript writing: All authors; (VII) Final approval of manuscript: All authors. \\ Correspondence to: Paola Ulivi. Biosciences Laboratory, Istituto Scientifico Romagnolo per lo Studio e la Cura dei Tumori (IRST) IRCCS, Via P. \\ Maroncelli 40, 47014 Meldola, Italy. Email: paola.ulivi@irst.emr.it.
}

Background: Molecular diagnostics for non-small cell lung cancer (NSCLC) has become the standard of care for personalized treatment. Epidermal growth factor receptor (EGFR) mutation and EML4-ALK translocation represent the two most important alterations in first-line treatment decision-making. However, other potentially targetable alterations are also present.

Methods: One thousand consecutive NSCLC patients with EGFR wild type (wt) tumors diagnosed by routine molecular analysis were considered. KRAS, BRAF, ERBB2, PIK3CA, NRAS, ALK, MAP2K1, RET and $D D R 2$ gene mutations were analyzed using the multiparametric Sequenom MassARRAY ${ }^{\circledR}$ platform. EML4$A L K$ and ROS1 rearrangements were also assessed by fluorescent in situ hybridization. HER4 status was determined by direct sequencing.

Results: Three hundred and forty-eight (34.8\%), 31 (3.1\%), 39 (4.4\%), $14(1.8 \%), 6(0.7 \%), 16(1.8 \%), 5$ $(0.6 \%)$ and $9(0.9 \%)$ patients showed an alteration in KRAS, BRAF, ALK, ROS1, NRAS, PIK3CA, MAPK1/2 and HER2 genes, respectively. Of the 657 patients for whom all markers were determined, 318 (48\%) patients had at least one alteration. Eight patients showed overlapping mutations, 4 KRAS mutation/EML4$A L K$ translocation, one KRAS mutation/ROS1 rearrangement, 2 KRAS/PIK3CA mutations, and one BRAF/ PIK3CA mutations.

Conclusions: About $50 \%$ of our patients had a potentially targetable alteration, confirming the usefulness of a multiparametric approach for routine molecular diagnostics aimed at identifying potential therapeutic targets.

Keywords: Non-small cell lung cancer (NSCLC); targeted therapy; epidermal growth factor receptor (EGFR); multitarget analysis; Formalin-fixed paraffin-embedded samples (FFPE samples)

Submitted Apr 11, 2018. Accepted for publication Jun 15, 2018.

doi: $10.21037 /$ jtd.2018.07.22

View this article at: http://dx.doi.org/10.21037/jtd.2018.07.22 


\section{Introduction}

Targeted therapy for non-small cell lung cancer (NSCLC) has transformed the outcome of patients carrying specific molecular alterations. In particular, epidermal growth factor receptor tyrosine kinase inhibitors (EGFR-TKIs), such as gefitinib, erlotinib or afatinib, and anti-ALK agents, such as crizotinib, have changed the natural history of adenocarcinoma patients carrying specific EGFR mutations or EML4-ALK translocation/ROS1 rearrangements, respectively (1-5). Other potentially targetable alterations have been identified in lung cancer. Of these, $B R A F$ and HER 2 mutations are present in about $3 \%$ and $2 \%$ of patients with lung adenocarcinoma, respectively (6-8) and represent possible targets for therapy using anti BRAF (vemurafenib or dabrafenib) or anti-HER2 (trastuzumab, dacomitinib, etc.) agents (8-11). Moreover, mesenchymalepithelial transition factor (MET) alterations (mutation or amplification) would seem to identify a subset of patients who are more likely to respond to crizotinib $(12,13)$. In addition, other potentially targetable alterations have been found in several genes, including NTRK1, PIK3CA, HER4, $N R A S$ (6,14-18), and the frequency of these alterations differs in different ethnicities. The number of clinical trials aimed at analyzing the effect of targeted drugs specific for these different alterations is thus expected to increase enormously in the near future.

In the present study we evaluated a large Italian cohort of NSCLC patients, all EGFR wild type (wt) according to diagnostic molecular analysis, to verify the frequency of potentially targetable alterations in relation to clinical pathological characteristics of patients.

\section{Methods}

\section{Patients}

We evaluated a cohort of 1,000 patients, all recruited from the Wide Catchment Area of Romagna (AVR), with histologically or cytologically confirmed advanced NSCLC classified as EGFR wt by routine diagnostic molecular analysis from January 2013 to December 2016. The clinical pathological characteristics of patients are reported in Table 1 . The study was approved by our institutional Review Board and all patients gave written informed consent.

\section{Biological samples}

Formalin-fixed paraffin-embedded (FFPE) histological samples, cytological FFPEs (cell blocks) or cytological smears were available for molecular analysis. Biological samples were evaluated and selected by AVR pathologists. Tumor specimens comprising at least $50 \%$ tumor cells were selected and underwent DNA extraction.

\section{EML4-ALK and ROS1 determinations}

Selected FFPE histological or cytological sections and cytological samples were used to perform EML4-ALK and ROS1 determinations. FISH assay was performed using a break-apart ALK or ROS1 probe (Vysis LSI Dual Color, Break Apart Rearrangement Probe; Abbott/Vysis, Illinois, IL, USA. ALK and ROS1 rearrangements were scored as positive when $\geq 15 \%$ of tumor cells displayed split signals or isolated signals containing a kinase domain (red for $A L K$ and green for ROS1), as previously described $(19,20)$. Slides containing at least 50 tumor cells were considered evaluable and were read independently by two experts blinded to the patient's history and histological findings.

\section{Mutation analysis}

Mutation analyses were centralized and performed in the Biosciences Laboratory of IRST IRCCS. KRAS, BRAF, ERBB2, PIK3CA, NRAS, ALK, MAP2K1, RET and DDR2 gene status was analyzed by Myriapod ${ }^{\circledR}$ Lung Status kit (Diatech Pharmacogenetics, Jesi, Italy) on MassARRAY ${ }^{\circledR}$ (SEQUENOM ${ }^{\circledR}$ Inc., San Diego, CA, USA). Exons 18 to 23 of the HER4 gene were evaluated by direct sequencing.

\section{Statistical analysis}

The chi-square test was used for group comparison of variables.

\section{Results}

\section{Frequency of gene alterations}

KRAS, BRAF and HER2 determinations were performed in the entire case series. Conversely, there was only sufficient biological to perform NRAS, PIK3CA, MAP2K1, ALK, RET and DDR2 mutation analysis in 901 patients, EML4-ALK evaluation in 889 patients and ROS1 determinations in 733 patients. Overall, characterization of all 11 markers was performed in 657 patients. HER4 mutation analysis was also carried out in 450 cases. Three hundred and forty-eight 
Table 1 Relation between the Different Alterations and the Clinical Pathological Characteristics of Patients

\begin{tabular}{|c|c|c|c|c|c|c|c|c|c|c|c|}
\hline $\begin{array}{l}\text { Gene } \\
\text { alteration }\end{array}$ & $\begin{array}{l}\text { Total No. } \\
\text { gene } \\
\text { mutations }\end{array}$ & \multicolumn{3}{|c|}{ Gender (\%) } & \multicolumn{3}{|c|}{ Age, years (\%) } & \multicolumn{4}{|c|}{ Smoking habits* No. (\%) } \\
\hline$B R A F$ & 31 & $13(41.9)$ & $18(58.1)$ & 0.633 & $17(54.8)$ & $14(45.2)$ & 0.669 & $3(15.0)$ & $6(30.0)$ & $11(55.0)$ & 0.955 \\
\hline NRAS & 6 & $4(67.0)$ & $2(33.0)$ & 0.204 & $6(100.0)$ & - & 0.032 & - & $2(50.0)$ & $2(50.0)$ & 0.656 \\
\hline MAPK $1 / 2$ & 5 & - & $5(100.0)$ & 0.164 & $4(80.0)$ & $1(20.0)$ & 0.376 & $1(20.0)$ & $1(20.0)$ & $3(60.0)$ & 1.000 \\
\hline HER2 & 9 & $6(66.7)$ & $3(33.3)$ & 0.087 & $4(44.0)$ & $5(56.0)$ & 0.746 & $2(40.0)$ & $2(40.0)$ & $1(20.0)$ & 0.221 \\
\hline EML4-ALK & 39 & $23(59.0)$ & $16(41.0)$ & 0.005 & $28(71.8)$ & $11(28.2)$ & 0.015 & $17(51.5)$ & $4(12.1)$ & $12(36.4)$ & $<0.001$ \\
\hline ROS1 & 14 & $9(64.3)$ & $5(35.7)$ & 0.053 & $8(57.1)$ & $6(42.9)$ & 0.748 & $6(66.7)$ & $1(11.1)$ & $2(22.2)$ & 0.002 \\
\hline
\end{tabular}

${ }^{*}$, percentages refer to the total number of patients with smoking habits information available.
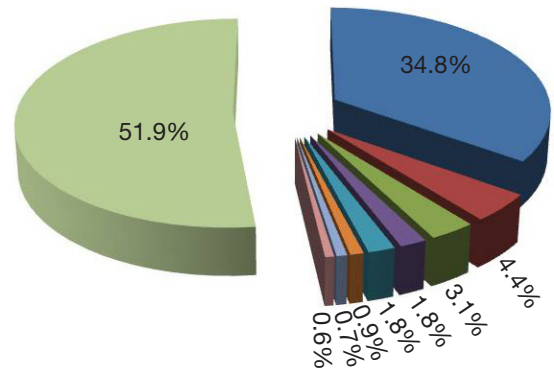

- KRAS

- EML4-ALK

$=B R A F$

- ROS1

- PIK3CA

- HER2

- NRAS

MAPK1/2

No oncogenic driving

Figure 1 Frequency of gene alterations in the entire case series of EGFR wt patients.

(34.8\%), 31 (3.1\%), 39 (4.4\%), 14 (1.8\%), 6 (0.7\%), 16 (1.8\%), 5 (0.6\%) and $9(0.9 \%)$ patients showed an alteration in KRAS, BRAF, ALK, ROS1, NRAS, PIK3CA, MAPK1/2 and HER2 genes, respectively (Figure 1). Of the 657 patients in whom all the markers were determined, 318 (48\%) showed at least one alteration. The different mutations found for each gene are shown in Figure 2. Eighty-four percent of KRAS mutations were found at codon 12, the majority (39\%) being G12C alterations, while $10.3 \%$ of mutations involved codon 13. Around half of all BRAF mutations (54.8\%) were V600E, whereas $45.2 \%$ were other exon 15 alterations or exon 11 mutations. In particular, 2 (14\%) of the mutated patients with no V600E alteration harbored a different exon 15 mutation (D594G), while 12 $(86 \%)$ showed an exon 11 alteration, 5 involving codon 466 (2 G466A, 2 G466E, one G466V) and 7 codon 469 (3 G469A, 1 G469E, 3 G469V). All NRAS mutations were at codon 61 (3 Q61K, 2 Q61L and one Q61R), whereas
PIK3CA alterations were found in exon $9(93.8 \%)$ in all but one patient (exon 20). Of the 5 patients carrying a MAPK1/2 mutation, 2 (40\%) had a Q56P alteration whereas 3 (60\%) showed a K57N substitution (Figure 2). Finally, all HER2-mutated patients had an exon 20 insertion. The only mutation found in HER4 gene was found in a former male smoker and located in exon $19(\mathrm{G} 735 \mathrm{~V})$. No alterations were found in $A L K$, RET or DDR2 genes.

Eight patients showed overlapping mutations: concomitant KRAS mutation and EML4-ALK translocation (4 cases); KRAS mutation together with ROS 1 rearrangement (1 case); concomitant KRAS and PIK3CA mutation ( 2 cases); and concomitant BRAF and PIK3CA mutation (1 case).

\section{Gene alterations in relation to clinical pathological characteristics of patients}

The relation between the different alterations and the clinical pathological characteristics of patients is reported in Table 2. EML4-ALK translocation was significantly correlated with gender, age and smoking habits $(\mathrm{P}=0.005$, $\mathrm{P}=0.015$ and $\mathrm{P}<0.001$, respectively) and was more frequent in young, non-smoking females. ROS1 rearrangements were significantly correlated with gender and smoking habits $(\mathrm{P}=0.053$ and $\mathrm{P}=0.002$, respectively) but not with age. Moreover, KRAS mutations were significantly more common in current smokers $(\mathrm{P}<0.001)$, whereas NRAS mutations were only found in patients $<70$ years of age $(\mathrm{P}=0.032)$.

Of the 4 patients showing concomitant EML4-ALK 
KRAS

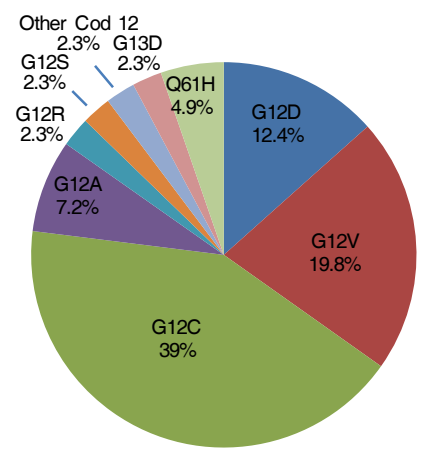

NRAS

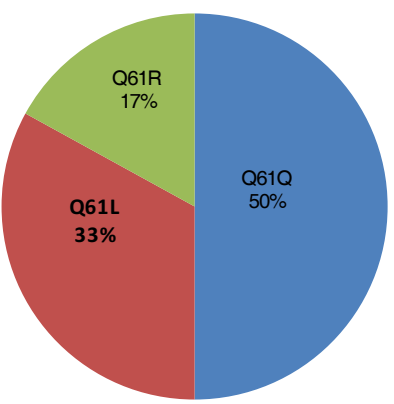

BRAF
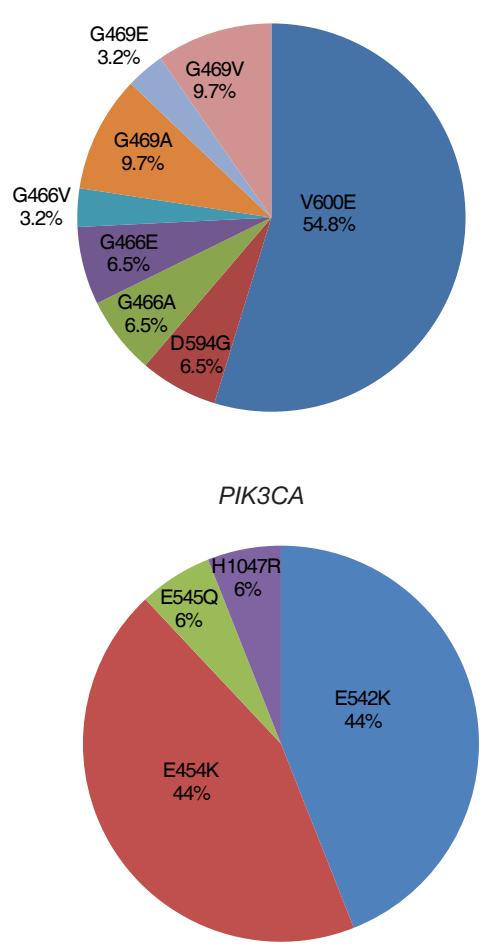

MAPK $1 / 2$

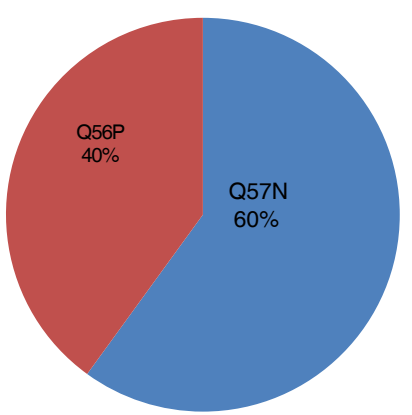

Figure 2 Types of mutations found in the different genes.

translocations and KRAS mutations ( 3 of whom were smokers), 2 were treated with first-line crizotinib and second-line ceritinib. One patient harboring a G12D KRAS mutation and with $70 \%$ fluorescent in situ hybridization (FISH) positivity initially showed stable disease with crizotinib but progressed after 5 cycles, and then again obtained stable disease with ceritinib, relapsing after 4 treatment cycles. Another patient with a G13S KRAS mutation and $50 \%$ FISH positivity obtained a partial response with crizotinib lasting 6 treatment cycles and another partial response with ceritinib lasting 3 cycles.
The only patient showing a concomitant KRAS mutation $(\mathrm{G} 12 \mathrm{~V})$ and ROS1 rearrangement was treated with secondline crizotinib but developed severe toxicity that led to treatment suspension before the clinical response could be evaluated.

\section{Discussion}

In the present study we report our results on a cohort of 1,000 consecutive NSCLC patients identified as EGFR wt by routine diagnostic molecular analysis performed at our 
Table 2 Clinical pathological characteristics of analyzed samples

\begin{tabular}{lc}
\hline Variables & No. (\%) \\
\hline Overall & 1,000 \\
Age, years & \\
$>70$ & $431(43.0)$ \\
$\leq 70$ & $569(57.0)$ \\
Gender & \\
Male & $622(62.0)$ \\
Female & $378(38.0)$ \\
Smoking habits & \\
Current & $379(37.9)$ \\
Former & $239(23.9)$ \\
Never & $145(14.5)$ \\
Missing & $237(23.7)$ \\
Histotype & \\
ADC & $793(79.3)$ \\
PDC & $178(17.8)$ \\
Other & $29(2.9)$ \\
Type of sample & \\
Histological & \\
\hline Cytological & \\
\hline
\end{tabular}

ADC, adenocarcinoma; PDC, poorly differentiated carcinoma.

institute (IRST IRCCS). We demonstrated that about half of all the EGFR wt patients carried a potentially targetable gene alteration. The frequencies of alterations were as follows: KRAS, 35\%; EML4-ALK, 4.4\%; BRAF, 3.1\%; ROS1, 0.7\%; PIK3CA, 1.8; NRAS, 1.8\%; MAPK1/2, 0.6\%; and HER2, $0.9 \%$. Such findings are in agreement with literature data $(6,15,16,21)$. A slightly higher frequency of $K R A S$ mutations was observed, possibly attributable to the fact that we considered a selected case series of $E G F R$ wt patients in whom KRAS mutations were more frequent due to the mutual exclusivity of the 2 gene mutations. In accordance with previous authors (22), we observed a higher incidence of KRAS mutation and a high prevalence of $\mathrm{G} 12 \mathrm{C}$ alterations in current smokers.

With regard to $B R A F$ mutation, we saw that almost half of the mutated patients carried non-V600E alterations that were predominantly located in exon 11 at codons 466 and 469. It is known that V600 alterations predict sensitivity to
anti-BRAF and anti-MEK combinations (23). Moreover, recent evidence suggests that non-V600 alterations may also be associated with sensitivity to such treatments (24). These results suggest that about $3 \%$ of EGFR wt patients could benefit from this type of targeted treatment. In agreement with other studies (7), no associations were observed between $B R A F$ mutations and clinical pathological characteristics of patients. Around $6 \%$ of our patients harbored an EML4-ALK (4.4\%) or ROS1 (1.8\%) rearrangement, the majority of whom were predominantly young females who had never smoked, as described in other studies $(19,25)$.

Other alterations that are potential targets for treatment are present in lung adenocarcinoma, e.g., $1.8 \%$ of our patients carried a PIK3CA mutation. Although there are still no drugs capable of inhibiting the growth of PIK3CA mutated cells, such mutations would seem to confer resistance to TKIs $(26,27)$, making their determination of clinical importance.

We observed a slightly lower percentage of HER2 mutations with respect to that described in the literature (28) but similar (1.7\%) to the findings of Mazières et al. (8). in a large case series. However, the relatively low sensitivity of the Sanger sequencing we used for the detection of HER2 mutations may partly explain our results. No significant associations were found between HER2 mutation and gender, age or smoking habits.

We also observed 8 patients with overlapping mutations, 4 of whom showed concomitant EML4-ALK and KRAS alterations. Of these, 2 underwent treatment with anti-ALK agents, one obtaining a partial response. In agreement with other authors, we have already seen that the presence of a $K R A S$ mutation in patients with EML4-ALK translocation can confer resistance to treatment with crizotinib $(29,30)$. Although the low number of double-mutated patients in our study does not permit us to draw any definitive conclusions about this, there were seem to be sufficient evidence to warrant KRAS status being taken into consideration in EML4-ALK translocated patients treated with anti-ALK agents.

In conclusion, although the frequency of single gene alterations in our study was low, about half of the patients with $E G F R$ wt lung adenocarcinoma analyzed showed a potentially targetable alteration. Anti-ALK agents have already been approved for use as first-line treatment of EML4-ALK-translocated tumors. However, larger, randomized clinical trials are needed to verify the usefulness of targeted agents in tumors harboring other specific alterations. 


\section{Acknowledgements}

The authors would like to thank Cristiano Verna for editorial assistance.

\section{Footnote}

Conflicts of Interest: The authors have no conflicts of interest to declare.

Ethical Statement: The study was approved by our institutional Review Board (No. 675 of 3.09.2013) and all patients gave written informed consent.

\section{References}

1. Mok TS, Wu YL, Thongprasert S, et al. Gefitinib or carboplatin-paclitaxel in pulmonary adenocarcinoma. $\mathrm{N}$ Engl J Med 2009;361:947-57.

2. Rosell R, Carcereny E, Gervais R, et al. Erlotinib versus standard chemotherapy as first-line treatment for European patients with advanced EGFR mutation-positive non-small-cell lung cancer (EURTAC): a multicentre, open-label, randomised phase 3 trial. Lancet Oncol 2012;13:239-46.

3. Yang JC, $\mathrm{Wu}$ YL, Schuler M, et al. Afatinib versus cisplatin-based chemotherapy for EGFR mutation-positive lung adenocarcinoma (LUX-Lung 3 and LUX-Lung 6): analysis of overall survival data from two randomised, phase 3 trials. Lancet Oncol 2015;16:141-51.

4. Solomon BJ, Mok T, Kim DW, et al. First-line crizotinib versus chemotherapy in ALK-positive lung cancer. N Engl J Med 2014;371:2167-77.

5. Mazières J, Zalcman G, Crinò L, et al. Crizotinib therapy for advanced lung adenocarcinoma and a ROS1 rearrangement: results from the EUROS1 cohort. J Clin Oncol 2015;33:992-9.

6. Kris MG, Johnson BE, Berry LD, et al. Using multiplexed assays of oncogenic drivers in lung cancers to select targeted drugs. JAMA 2014;311:1998-2006.

7. Chen D, Zhang LQ, Huang JF, et al. BRAF mutations in patients with non-small cell lung cancer: a systematic review and meta-analysis. PLoS One 2014;9:e101354.

8. Mazières J, Peters S, Lepage B, et al. Lung cancer that harbors an HER2 mutation: epidemiologic characteristics and therapeutic perspectives. J Clin Oncol 2013;31:1997-2003.

9. Gautschi O, Milia J, Cabarrou B, et al. Targeted therapy for patients with BRAF-mutant lung cancer: results from the european EURAF cohort. J Thorac Oncol 2015;10:1451-7.

10. Hyman DM, Puzanov I, Subbiah V, et al. Vemurafenib in multiple nonmelanoma cancers with BRAF V600 mutations. N Engl J Med 2015;373:726-36.

11. Kris MG, Camidge DR, Giaccone G, et al. Targeting HER2 aberrations as actionable drivers in lung cancers: phase II trial of the pan-HER tyrosine kinase inhibitor dacomitinib in patients with HER2-mutant or amplified tumors. Ann Oncol 2015;26:1421-7.

12. Lu X, Peled N, Greer J, et al. MET Exon 14 mutation encodes an actionable therapeutic target in lung adenocarcinoma. Cancer Res 2017;77:4498-505.

13. Suryavanshi M, Shah A, Kumar D, et al. MET amplification and response to MET inhibitors in stage IV lung adenocarcinoma. Oncol Res Treat 2017;40:198-202.

14. Ding L, Getz G, Wheeler DA, et al. Somatic mutations affect key pathways in lung adenocarcinoma. Nature 2008;455:1069-75.

15. Sholl LM, Aisner DL, Varella-Garcia M, et al. Multiinstitutional oncogenic driver mutation analysis in lung adenocarcinoma: the Lung Cancer Mutation Consortium experience. J Thorac Oncol 2015;10:768-77.

16. Yeung SF, Tong JH, Law PP, et al. Profiling of oncogenic driver events in lung adenocarcinoma revealed MET mutation as independent prognostic factor. J Thorac Oncol 2015;10:1292-300.

17. Schrock AB, Frampton GM, Suh J, et al. Characterization of 298 patients with lung cancer harboring MET exon 14 skipping alterations. J Thorac Oncol 2016;11:1493-1502.

18. Vaishnavi A, Capelletti M, Le AT, et al. Oncogenic and drug-sensitive NTRK1 rearrangements in lung cancer. Nat Med 2013;19:1469-72.

19. Bergethon K, Shaw AT, Ou SH, et al. ROS1 rearrangements define a unique molecular class of lung cancers. J Clin Oncol 2012;30:863-70.

20. Kwak EL, Bang YJ, Camidge DR, et al. Anaplastic lymphoma kinase inhibition in non-small-cell lung cancer. N Engl J Med 2010;363:1693-703.

21. Daoud A, Chu QS. Targeting novel but less common driver mutations and chromosomal translocations in advanced non-small cell lung cancer. Front Oncol 2017;7:222.

22. Chapman AM, Sun KY, Ruestow P, et al. Lung cancer mutation profile of EGFR, ALK, and KRAS: meta-analysis and comparison of never and ever smokers. Lung Cancer 2016;102:122-34. 
23. Planchard D, Smit EF, Groen HJ, et al. Dabrafenib plus trametinib in patients with previously untreated BRAFV600E-mutant metastatic non-small-cell lung cancer: an open-label, phase 2 trial. Lancet Oncol 2017;18:1307-16.

24. Noeparast A, Teugels E, Giron P, et al. Non-V600 BRAF mutations recurrently found in lung cancer predict sensitivity to the combination of Trametinib and Dabrafenib. Oncotarget 2016;8:60094-108.

25. Pillai RN, Ramalingam SS. The biology and clinical features of non-small cell lung cancers with EML4-ALK translocation. Curr Oncol Rep 2012;14:105-10.

26. Chen JY, Cheng YN, Han L, et al. Predictive value of K-ras and PIK3CA in non-small cell lung cancer patients treated with EGFR-TKIs: a systemic review and meta-analysis. Cancer Biol Med 2015;12:126-39.

27. Wu SG, Chang YL, Yu CJ, et al. The Role of PIK3CA

Cite this article as: Chiadini E, Canale M, Delmonte A, Dazzi C, Casanova C, Capelli L, Mariotti M, Papi M, Gamboni A, Puccetti M, Bravaccini S, Dubini A, Calistri D, Crinò L, Ulivi P. Frequency of actionable alterations in EGFR wild type nonsmall cell lung cancer: experience of the Wide Catchment Area of Romagna (AVR). J Thorac Dis 2018;10(8):4858-4864. doi: 10.21037/jtd.2018.07.22 mutations among lung adenocarcinoma patients with primary and acquired resistance to EGFR tyrosine kinase inhibition. Sci Rep 2016;6:35249.

28. Pillai RN, Behera M, Berry LD, et al. HER2 mutations in lung adenocarcinomas: a report from the Lung Cancer Mutation Consortium. Cancer 2017;123:4099-105.

29. Ulivi P, Chiadini E, Dazzi C, et al. Nonsquamous, non-small-cell lung cancer patients who carry a double mutation of EGFR, EML4-ALK or KRAS: frequency, clinical-pathological characteristics, and response to therapy. Clin Lung Cancer 2016;17:384-90.

30. Mengoli MC, Barbieri F, Bertolini F, et al. K-RAS mutations indicating primary resistance to crizotinib in ALK-rearranged adenocarcinomas of the lung: report of two cases and review of the literature. Lung Cancer 2016;93:55-8. 